\title{
Kinetic Study on Esterification of Acetic Acid with Isopropyl Alcohol Catalyzed by Ion Exchange Resin
}

\author{
Ying Liu, ${ }^{\circledR}$ Jia Liu, Hanzhao Yan, Zheng Zhou,** and Aidong Zhou \\ School of Chemistry and Chemical Engineering, Nanjing University, 210023 Nanjing, China \\ Supporting Information
}

ABSTRACT: Esterification kinetics on acetic acid with isopropyl alcohol was studied in an intensified fixed bed reactor at $333-353 \mathrm{~K}$ with Amberlyst 36 Wet. The effects of volume flow rate, molar ratio of reactants, catalyst loading, and operating temperature were investigated and optimized. The method of UNIFAC was applied to calculate the activity coefficient of each component for correcting the nonideality of the solution. Reaction enthalpy, entropy, and Gibbs free energy were calculated in different cases. The pseudohomogeneous model, EleyRideal model, and Langmuir-Hinshelwood-Hougen-Watson model were used to establish kinetic equations of the reaction conducted in the IFBR. It was proved that the LHHW model can accurately describe the esterification kinetics in the intensified fixed bed reactor.

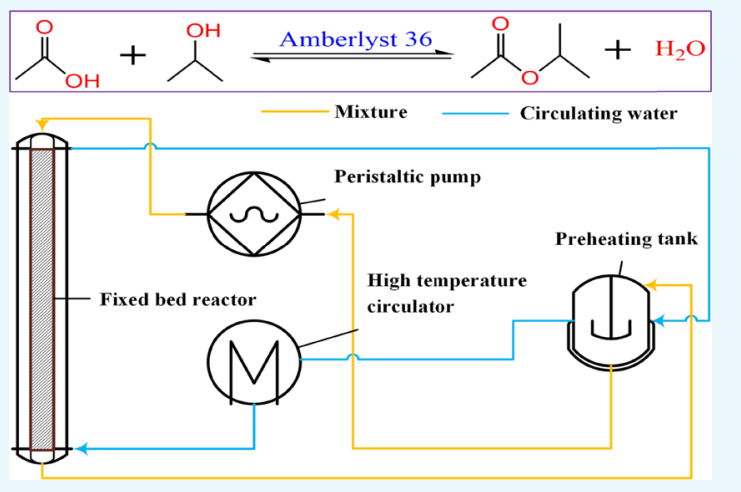

\section{INTRODUCTION}

Isopropyl acetate (IPAc) is a kind of important fine chemical intermediate, which is known as the universal solvent. It can be used in high-class ink oil, pharmaceuticals, pesticide, adhesive, dehydrating agent, and extractant. IPAc also can be found in fragrance composition. ${ }^{1-3}$

IPAc can be synthetized by esterification of acetic acid (HAc) with isopropyl alcohol (IPA) in the existence of ion exchange resin catalysts. Also, many kinetic studies on esterification based on ion exchange resin catalysts have been reported before. Altıokka and Çıtak reported the kinetic characteristics of the esterification of HAc with isobutanol by Amberlite IR-120 with the Eley-Rideal(ER) model. ${ }^{4}$ The same catalyst was used by Osorio-Viana et al. to study esterification of HAc with isoamyl alcohol. ${ }^{5}$ The pseudohomogeneous( $\mathrm{PH})$ model, Langmuir-HinshelwoodHougen-Watson (LHHW) model, and two other models were applied to describe the reaction kinetics. Besides, JagadeeshBabu et al. studied esterification kinetics of HAc with methanol with different kinds of ion exchange catalysts (Indion 190, Indion 130, and Amberlyst 15 Wet). ${ }^{6}$ They found that Indion 130 was effective for HAc esterification and established kinetic equations with the LHHW model by supposing that the adsorption is slight for each component.

Kinetic studies reported mostly were conducted in stirred tanks ${ }^{4-8}$ or traditional fixed bed reactors. ${ }^{9-13}$ In this work, an intensified fixed bed reactor (IFBR), improved from the traditional fixed bed reactor by our research group, ${ }^{14}$ was applied to conduct esterification reactions of HAc with IPA in the existence of Amberlyst 36 Wet. In the traditional fixed bed reactor, the reactants flow freely from the top of the bed through the catalysts without circulation and are then discharged. However, the IFBR is equipped with a circulating pump to pass the reactants through the catalyst bed multiple times at a certain flow rate. Besides, the inner diameter of the fixed bed pipe is smaller than the traditional one. The linear speed of the fluid is heightened, and the liquid film is thinner after improvements, which significantly increases the reaction rate. In this paper, the kinetic equations based on the IFBR were established by the use of PH, ER, and LHHW models.

\section{RESULTS AND DISCUSSION}

2.1. Effects of Parameters. 2.1.1. Effect of Volume Flow Rate. Experiments on the volume flow rate were carried out for $5 \mathrm{~h}$ at $348.15 \mathrm{~K}$ with $15 \mathrm{wt} \%$ (percentage of raw material weight) catalyst loading and an initial HAc/IPA molar ratio of up to 1.5 , the result of which is shown in Figure 1. The result shows that the reaction rate is increased when the circulating flow rate rises from 20 to $40 \mathrm{~mL} / \mathrm{min}$ and then basically unchanged when the circulating flow rate increases from 40 to $100 \mathrm{~mL} / \mathrm{min}$. This indicates that the reaction rate is unaffected by the external diffusion when the flow rate exceeds $40 \mathrm{~mL}$ / min. Chakrabarti and Sharma ${ }^{15}$ show that the rate of the reaction process catalyzed by ion exchange resin will not be controlled by the external diffusion unless the viscosity of the reactants is especially high or the speed of agitation (volume flow rate here) is very low. Therefore, the flow rate should be maintained above $40 \mathrm{~mL} / \mathrm{min}$ to make certain of the removal of the effects of external diffusion and enhanced space velocity.

2.1.2. Effect of Molar Ratio. To investigate the influence of molar ratio, the molar ratio of HAc and IPA was changed from $1: 1$ to $2.5: 1$, while other reaction conditions remained the

Received: September 13, 2019

Accepted: October 25, 2019

Published: November 7, 2019 


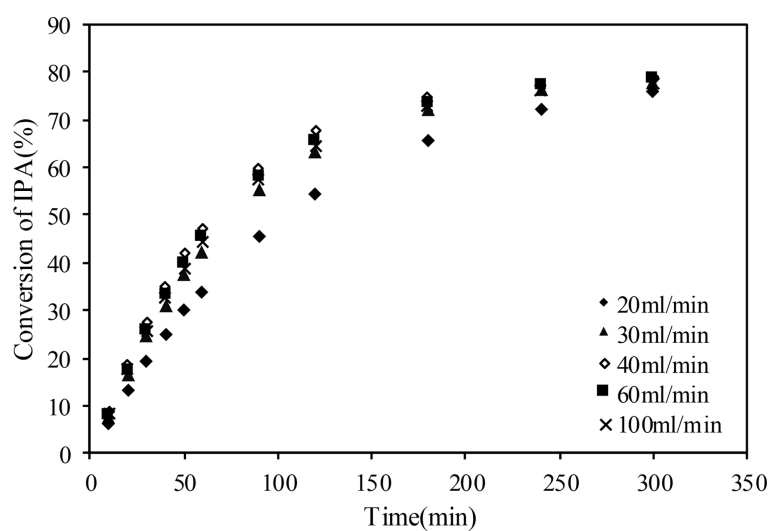

Figure 1. Effect of volume flow rate on conversion of IPA. Temperature, $348.15 \mathrm{~K}$; initial molar ratio of HAc and IPA, 1.5; catalyst loading, 15 wt $\%$.

same. The experiments were based on the fact that the total mass of mixture was unchanged. The result is shown in Figure 2. It is clearly shown that the conversion of IPA goes up from

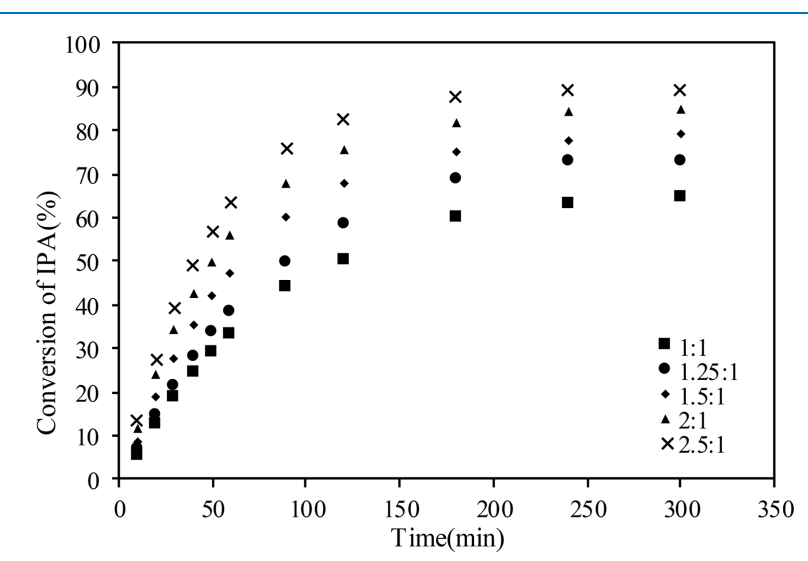

Figure 2. Effect of molar ratio HAc/IPA on conversion of IPA. Temperature, $348.15 \mathrm{~K}$; volume flow rate, $40 \mathrm{~mL} / \mathrm{min}$; catalyst loading, 15 wt $\%$.

65.1 to $89.5 \%$ as the HAc/IPA molar ratio increases from 1:1 to $2.5: 1$. Unchanged total mass means that the concentration of HAc goes up and that of IPA decreases, which lead to the shift of reaction equilibrium toward the products.

2.1.3. Effect of Catalyst Loading. Amberlyst 36 Wet was adopted to investigate the appropriate catalyst loading and the effect of that on the conversion of IPA. Catalyst loading was varied from 5 to $25 \mathrm{wt} \%$ at $348.15 \mathrm{~K}$ with an initial HAc/IPA molar ratio of up to 1.5 and a volume flow rate staying at 40 $\mathrm{mL} / \mathrm{min}$. Figure 3 clearly shows that the conversion of IPA rises from 45.4 to $79.5 \%$ with catalyst loading from 5 to $20 \mathrm{wt}$ $\%$ at $180 \mathrm{~min}$, while that of IPA only increases from 79.5 to $82.2 \%$ with catalyst loading from 20 to 25 wt \%. This indicates a large increase in the reaction rate as catalyst loading goes up to $20 \mathrm{wt} \%$, which is attributed to more $\mathrm{H}^{+}$active centers available. The reaction rate does not increase much as catalyst loading goes up from 20 to 25 wt \%, which means that the number of active centers reaches saturation. Thus, 20-25 wt \% catalyst loading is more suitable under the reaction conditions.

2.1.4. Effect of Reaction Temperature. Esterification equilibrium is closely related to reaction temperature, which can be further studied by achieving the reaction equilibrium constant at different temperatures. Experiments on reaction

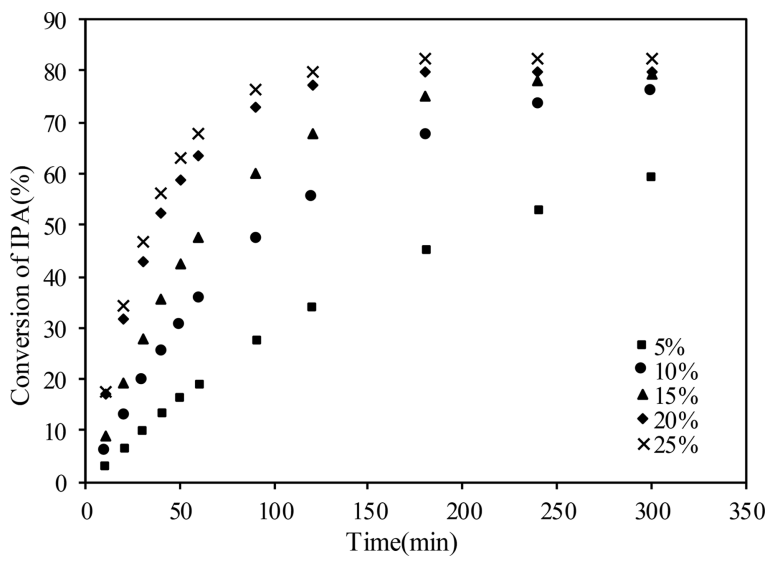

Figure 3. Effect of catalyst loading on conversion of IPA. Temperature, $348.15 \mathrm{~K}$; volume flow rate, $40 \mathrm{~mL} / \mathrm{min}$; initial molar ratio of HAc and IPA, 1.5.

temperature were conducted from 333.15 to $353.15 \mathrm{~K}$ with 25 wt $\%$ catalyst loading and other invariant conditions, the result of which is shown in Figure 4. As shown in the figure, the

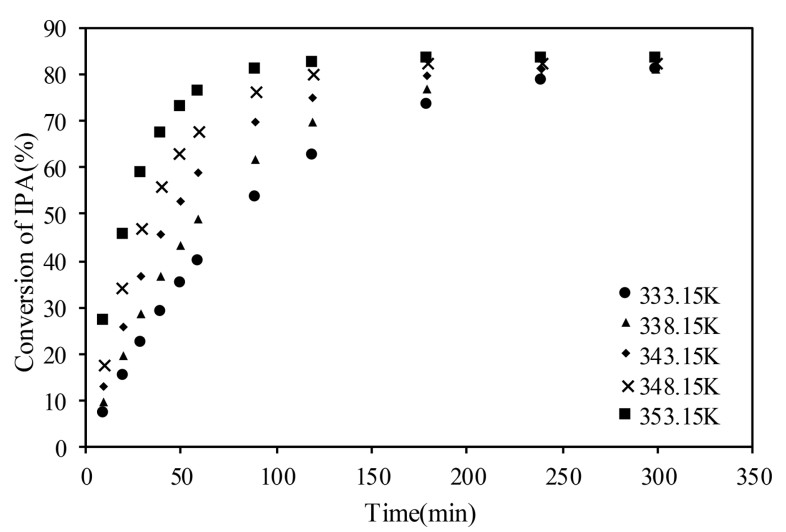

Figure 4. Effect of reaction temperature on conversion of IPA. Catalyst loading, $25 \mathrm{wt} \%$; volume flow rate, $40 \mathrm{~mL} / \mathrm{min}$; initial molar ratio of HAc and IPA, 1.5.

reaction rate significantly increases with increasing reaction temperature, which indicates that the chemical reaction process is a rate-controlling step. ${ }^{8}$ In addition, final conversion of IPA at different temperatures has little difference, which proves that the reaction temperature has little influence on the final conversion of IPA. This phenomenon is consistent with the conclusion obtained below that esterification of IPA with HAc is an extremely mild exothermic reaction, the heat of reaction of which is $-2.37 \mathrm{~kJ} / \mathrm{mol}$.

2.2. Thermodynamic Parameters. 2.2.1. Equilibrium Constant. The equilibrium constant $K_{\mathrm{a}}$ was determined by experiments conducted at different temperatures ranging from 333.15 to $353.15 \mathrm{~K}$. Considering the nonideality of the mixed solution, the equilibrium constant was calculated by activity of each component at equilibrium instead of concentration. Thus, expression of $K_{\mathrm{a}}$ based on the activity of components is given in eq 1.

$$
K_{\mathrm{a}}=\frac{a_{\mathrm{IPAc}} a_{\mathrm{H}_{2} \mathrm{O}}}{a_{\mathrm{IPA}} a_{\mathrm{HAc}}}=\left(\frac{x_{\mathrm{IPAc}} x_{\mathrm{H}_{2} \mathrm{O}}}{x_{\mathrm{IPA}} x_{\mathrm{HAc}}}\right)\left(\frac{\gamma_{\mathrm{IPAc}} \gamma_{\mathrm{H}_{2} \mathrm{O}}}{\gamma_{\mathrm{IPA}} \gamma_{\mathrm{HAc}}}\right)
$$


where $a_{i}, x_{i}$, and $\gamma_{i}$, respectively, are the activity, mole fraction, and activity coefficient of component $i$ at equilibrium. The mole fraction $x_{i}$ is determined by experiments, and the activity coefficient is estimated by the UNIFAC model. ${ }^{16}$ In the UNIFAC method, the activity coefficient is divided into a combined part, $\gamma_{i}^{(c)}$, and a residual part, $\gamma_{i}^{(R)}$, which is demonstrated in eq 2 .

$$
\ln \gamma_{i}=\ln \gamma_{i}^{(c)}+\ln \gamma_{i}^{(R)}
$$

where the combined part of the activity coefficient $\gamma_{i}^{\left({ }^{(c)}\right.}$ is calculated by the volume and area parameters of the groups given in Table S1 (Supporting Information).

The residual part $\gamma_{i}^{(R)}$ is calculated by the interaction parameters of the groups $a_{m n}$ given in Table S2. The specific calculation process is given by Poling et al. ${ }^{16}$ Calculation results of $\gamma_{i}$ and $K_{\mathrm{a}}$ are listed in Table 1 .

Table 1. Activity Coefficient of the Components and Equilibrium Constants at Different Temperatures

\begin{tabular}{llllll}
$T(\mathrm{~K})$ & 333.15 & 338.15 & 343.15 & 348.15 & 353.15 \\
\hline$\gamma_{\mathrm{HAc}}$ & 0.84045 & 0.84606 & 0.85171 & 0.85940 & 0.86560 \\
$\gamma_{\mathrm{IPA}}$ & 1.2915 & 1.2921 & 1.2930 & 1.2989 & 1.3025 \\
$\gamma_{\mathrm{IPAc}}$ & 1.7147 & 1.7099 & 1.7059 & 1.7096 & 1.7097 \\
$\gamma_{\mathrm{H}_{2} \mathrm{O}}$ & 2.3929 & 2.3867 & 2.3811 & 2.3799 & 2.3769 \\
$K_{\mathrm{a}}$ & 23.315 & 23.030 & 22.751 & 22.451 & 22.224 \\
\hline
\end{tabular}

Table 1 gives equilibrium constants at different temperatures calculated by eq 1 . It clearly shows that the value of $K_{a}$ decreases from 23.315 to 22.224 as the temperature increases from 333.15 to $353.15 \mathrm{~K}$, which shows that the reaction is exothermic.

2.2.2. $\Delta_{r} H^{0}, \Delta_{r} S^{0}$, and $\Delta_{r} G^{0}$ of Reaction. It is assumed that the reaction enthalpy $\Delta_{r} H^{0}$ and reaction entropy $\Delta_{r} S^{0}$ are both constants within the experimental temperature range. Thus, temperature dependence of the equilibrium constant is known from the chemical reaction isotherm equation (Van't Hoff equation) and the definition of Gibbs free energy, which is given in eq 3 .

$$
\ln K_{\mathrm{a}}=-\frac{\Delta_{r} G^{0}}{R T}=-\frac{\Delta_{r} H^{0}}{R T}+\frac{\Delta_{r} S^{0}}{R}
$$

Equation 3 obviously shows that $\ln K_{\mathrm{a}}$ is linear to $1 / T$. Therefore, $T$ dependence of $K_{\mathrm{a}}$ is given in Figure 5 by plotting $\ln K_{\mathrm{a}}$ against $1 / T$. From the slope and intercept in the figure,

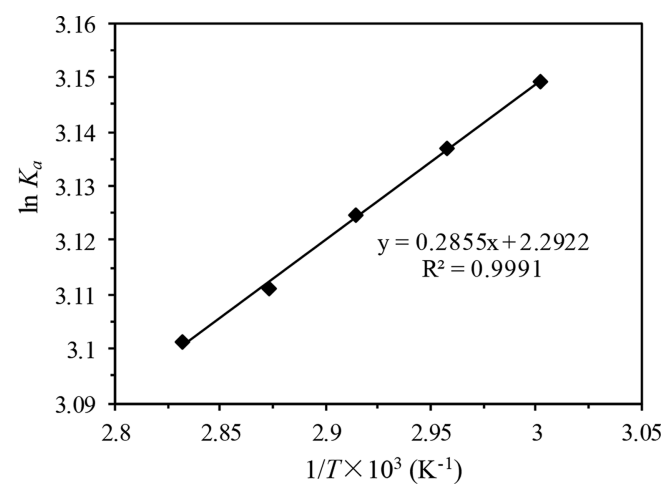

Figure 5. $T$ dependence of $K_{\mathrm{a}}$.
$\Delta_{r} H^{0}, \Delta_{r} S^{0}$, and $\Delta_{r} G^{0}$, respectively, are found to be $-2.37 \mathrm{~kJ} /$ $\mathrm{mol}, 19.06 \mathrm{~J} /(\mathrm{mol} \cdot \mathrm{K})$, and $-8.05 \mathrm{~kJ} / \mathrm{mol}(298.15 \mathrm{~K})$.

However, the actual reaction enthalpy varies with the temperature. The relationship between the two can be determined by the Kirchhoff equation

$$
\frac{\mathrm{d} \Delta_{r} H^{0}}{\mathrm{~d} T}=\sum_{i} v_{i} C_{p, i}
$$

where $C_{p, i}$ represents the molar heat capacity of component $i$, and $\nu_{i}$ are stoichiometric coefficients, which are positive for products and negative for reactants.

The method of Rowlinson-Bondi ${ }^{16}$ is adopted to get $C_{p, i}$ which is shown in eq 5

$$
\begin{aligned}
\frac{C_{p, i}-C_{p}^{0}}{R}= & 2.56+\frac{0.436}{1-T_{r}}+\omega\left[2.91+\frac{4.28\left(1-T_{r}\right)^{1 / 3}}{T_{r}}\right. \\
& \left.+\frac{0.293}{1-T_{r}}\right]
\end{aligned}
$$

where $T_{r}=T / T_{c}, T_{r}$ is the contrast temperature, $\omega$ is the acentric factor, $C_{p}^{0}$ is the heat capacity of an ideal gas at a given temperature, and $T_{c}$ is the critical temperature.

$T_{c}, C_{p}{ }^{0}$, and $\omega$ are calculated by the method of Constantinou-Gani, ${ }^{16}$ and then $C_{p, i}$ is obtained from eq 5 . $C_{p, i}$ is fitted to the third-order polynomial with temperature as an independent variable in eq 6 .

$$
C_{p, i}=a_{i}+b_{i} T+c_{i} T^{2}+d_{i} T^{3}
$$

where $a_{i}, b_{i}, c_{i}$, and $d_{i}$ are coefficients, which are given in Table 2 .

Table 2. Heat Capacity Coefficients of Each Component

\begin{tabular}{ccccc} 
component & $\begin{array}{c}a_{i} \\
(\mathrm{~J} /(\mathrm{mol} \cdot \mathrm{K}))\end{array}$ & $\begin{array}{c}b_{i} \\
\left.\mathrm{~J} /\left(\mathrm{mol} \cdot \mathrm{K}^{2}\right)\right)\end{array}$ & $\begin{array}{c}c_{i} \\
\left(\mathrm{~J} /\left(\mathrm{mol} \cdot \mathrm{K}^{3}\right)\right)\end{array}$ & $\begin{array}{c}d_{i} \\
\left(\mathrm{~J} /\left(\mathrm{mol} \cdot \mathrm{K}^{4}\right)\right)\end{array}$ \\
$\mathrm{HAc}$ & 160.23 & -0.301 & $8 \times 10^{-4}$ & $-4 \times 10^{-7}$ \\
$\mathrm{IPA}$ & 107.42 & 0.4907 & $-1.6 \times 10^{-3}$ & $2 \times 10^{-6}$ \\
$\mathrm{IPAc}$ & 120.69 & 0.0533 & $6 \times 10^{-4}$ & $-3 \times 10^{-7}$ \\
$\mathrm{H}_{2} \mathrm{O}$ & 151.26 & -0.3554 & $6 \times 10^{-4}$ & $-3 \times 10^{-7}$ \\
\hline
\end{tabular}

In addition, it is known from the Van't Hoff equation that

$$
\frac{\mathrm{d} \ln K_{\mathrm{a}}}{\mathrm{d} T}=\frac{\Delta_{r} H}{R T^{2}}
$$

The expression of $C_{p, i}$ is substituted into eqs 4 and 7 , and temperature dependence of $\Delta_{r} H, \Delta_{r} S, \Delta_{r} G$, and $K_{\mathrm{a}}$ are found to be as follows. ${ }^{17}$

$$
\begin{aligned}
& \Delta_{r} H=I_{K}+a T+\frac{b}{2} T^{2}+\frac{c}{3} T^{3}+\frac{d}{4} T^{4} \\
& \Delta_{r} S=R \cdot I_{H}+a(\ln T+1)+b T+\frac{c}{2} T^{2}+\frac{d}{3} T^{3} \\
& \Delta_{r} G=I_{K}-R \cdot I_{H} \cdot T-a T \ln T-\frac{b}{2} T^{2}-\frac{c}{6} T^{3}-\frac{d}{12} T^{4} \\
& \ln K_{\mathrm{a}}=I_{H}-\frac{I_{K}}{R T}+\frac{a}{R} \ln T+\frac{b}{2 R} T+\frac{c}{6 R} T^{2}+\frac{d}{12 R} T^{3}
\end{aligned}
$$


where $a=\sum_{i} v_{i} a_{i}, b=\sum_{i} v_{i} b_{i}, c=\sum_{i} v_{i} c_{i}, d=\sum_{i} v_{i} d_{i}$, and both $I_{K}$ and $R \cdot I_{H}$ are constant terms. Equation 11 can be transformed into eq 12

$$
\begin{aligned}
\ln & K_{\mathrm{a}}-\left(\frac{a}{R T} \ln T+\frac{b}{2 R} T+\frac{c}{6 R} T^{2}+\frac{d}{12 R} T^{3}\right) \\
= & \ln K_{\mathrm{a}}-f(T) \\
& =I_{H}-\frac{I_{K}}{R T}
\end{aligned}
$$

$T$ dependence of $\ln K_{\mathrm{a}}-f(T)$ is given in Figure 6 by plotting $\ln K_{\mathrm{a}}-f(T)$ against $1 / T$. From Figure 6, it can be seen that

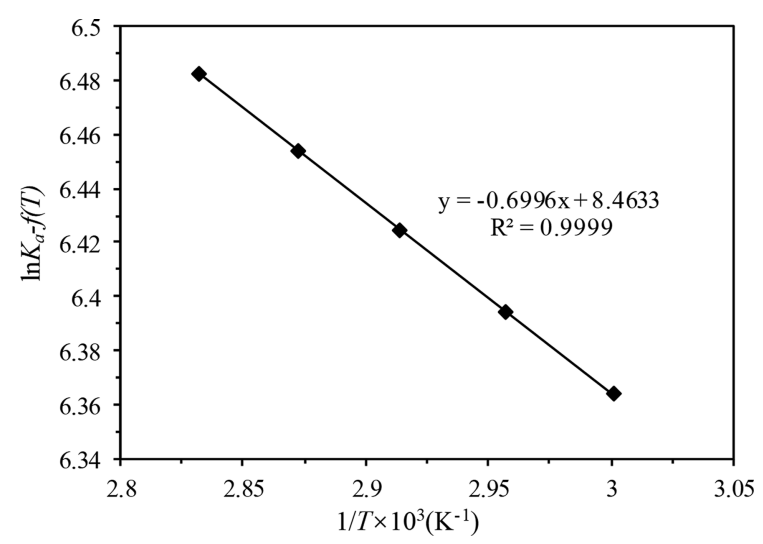

Figure 6. $T$ dependence of $\ln K_{\mathrm{a}}-f(T)$.

$$
\ln K_{\mathrm{a}}-f(T)=8.4633-\frac{699.6}{T}=8.4633-\frac{5816.47}{R T}
$$

where $I_{H}=8.4633$ and $I_{K}=5816.47 \mathrm{~J} / \mathrm{mol}$. The results obtained from eq 13 are substituted into eqs $8-10$, and the expressions of $\Delta_{r} H, \Delta_{r} S$, and $\Delta_{r} G$ on the temperature are gained. At $298.15 \mathrm{~K}, \Delta_{r} H^{0}, \Delta_{r} S^{0}$, and $\Delta_{r} G^{0}$, respectively, are found to be $-1.44 \mathrm{~kJ} / \mathrm{mol}, 21.99 \mathrm{~J} /(\mathrm{mol} \cdot \mathrm{K})$, and $-8.0 \mathrm{~kJ} / \mathrm{mol}$.

Finally, $\Delta_{r} H^{0}, \Delta_{r} S^{0}$, and $\Delta_{r} G^{0}$ obtained by different methods are listed in Table 3, from which it can be found that the results calculated by different methods have no significant

\begin{tabular}{|c|c|c|c|}
\hline condition & $\begin{array}{c}\Delta_{,} H^{0} \\
(\mathrm{~kJ} / \mathrm{mol})\end{array}$ & $\begin{array}{c}\Delta_{r} S^{0} \\
(\mathrm{~J} /(\mathrm{mol} \cdot \mathrm{K}))\end{array}$ & $\underset{(\mathrm{kJ} / \mathrm{mol})}{\Delta_{r} G^{0}}$ \\
\hline$\Delta_{r} H^{0}$ is the constant value & -2.37 & 19.06 & -8.05 \\
\hline$\Delta_{r} H^{0}$ changes with temperature & -1.44 & 21.99 & -8.0 \\
\hline
\end{tabular}
difference in numerical value.

Table 3. $\Delta_{r} H^{0}, \Delta_{r} S^{0}$, and $\Delta_{r} G^{0}$ of Esterification of HAc with IPA (at $298.15 \mathrm{~K}$ )

2.3. Reaction Kinetics. 2.3.1. External and Internal Diffusion Significance. According to the mass transfer theory, heightening the linear speed of the fluid can improve the turbulence of the fluid and make the liquid film thin enough that the resistance can be neglected. Thus, the external diffusion resistance can be reduced and even be small enough to be negligible. ${ }^{18}$ In the IFBR, the external diffusion is eliminated by increasing the circulating flow rate above 40 $\mathrm{mL} / \mathrm{min}$, which has been described before.

The effect of internal diffusion can be evaluated with the Weisz-Prater criterion $\left(C_{\mathrm{wp}}\right){ }^{18}$ which can be calculated with eq 14. If $C_{w p}$ is far less than 1 , effects of internal diffusion on the second-order reaction can be neglected.

$$
C_{\mathrm{wp}}=\frac{-r_{\mathrm{ob}} \rho_{\mathrm{p}} R_{\mathrm{c}}{ }^{2}}{D_{\mathrm{e}} C_{\mathrm{s}}}
$$

where $\rho_{\mathrm{p}}$ is the density of the catalyst, $-r_{\text {obs }}$ is the observed reaction rate, $R_{\mathrm{c}}$ is the ratio of the volume of catalyst pellet to the external surface area, $C_{s}$ is the reactant concentration at the catalyst surface, and $D_{\mathrm{e}}$ is the effective diffusion coefficient, ${ }^{19}$ which is calculated by eq 15 .

$$
D_{\mathrm{e}}=\frac{\varepsilon_{\mathrm{p}} D_{\mathrm{A}}}{\tau}
$$

where $\varepsilon_{\mathrm{p}}\left(\varepsilon_{\mathrm{p}}=0.24\right.$ here $)$ is the porosity; $\tau$, calculated by $\tau=$ $1 / \varepsilon_{\mathrm{p}}$, is the tortuosity factor; and $D_{\mathrm{A}}$ represents the infinite dilution diffusion coefficient, which is expressed by the method of Scheibel ${ }^{16}$

$$
D_{\mathrm{A}}=\frac{K T}{\mu_{2} V_{b 1}^{1 / 3}}
$$

where $K=17.5 \times 10^{-8}$ in the system of HAc and IPA, $\mu_{2}$ is the viscosity of solvent 2 , and $V_{b 1}$ represents the molar volume of solute 1 at its normal boiling temperature, which is calculated by the formula of Tyn-Calus.

The Weisz-Prater parameters calculated at different temperatures are given in Table S3. The values of $C_{\mathrm{wp}}$ are found in the range of 0.026 at $333.15 \mathrm{~K}$ to 0.071 at $353.15 \mathrm{~K}$, which are much less than 1 . This indicates that the influence of internal diffusion can be negligible in the reaction system.

2.3.2. Kinetic Model. The esterification of HAc with IPA can be represented by eq 17

$$
\mathrm{IPA}+\mathrm{HAc} \underset{k_{r}}{\stackrel{k_{f}}{\rightleftharpoons}} \mathrm{IPAc}+\mathrm{H}_{2} \mathrm{O}
$$

where $k_{f}$ and $k_{r}$, respectively, represent apparent rate constants of forward and reverse reactions. The pseudohomogeneous (PH) model, ${ }^{2,5-7,10}$ Eley-Rideal (ER) model, ${ }^{2,4,7,10}$ and Langmuir-Hinshelwood-Hougen-Watson (LHHW) $)^{2,5,7,8,10}$ model are applied to establish the kinetic equation of the reaction without external and internal diffusion limitations.

The PH model kinetic equation is given as follows

$$
\begin{aligned}
r_{\mathrm{PH}} & =M_{\mathrm{cat}}\left(k_{f} a_{\mathrm{HAc}} a_{\mathrm{IPA}}-k_{r} a_{\mathrm{IPAc}} a_{\mathrm{H}_{2} \mathrm{O}}\right) \\
& =M_{\mathrm{cat}} k_{f}\left(a_{\mathrm{HAc}} a_{\mathrm{IPA}}-\frac{1}{K_{\mathrm{a}}} a_{\mathrm{IPAc}} a_{\mathrm{H}_{2} \mathrm{O}}\right)
\end{aligned}
$$

where $M_{\text {cat }}$ represents the mass of catalyst per unit volume of the reactants, and $k_{r}=k_{f} / K_{\mathrm{a}}$.

The ER model supposes that the reactant molecule adsorbed on the active sites of the catalysts reacts with the other one in bulk solution. The kinetic equation is given as follows

$$
\begin{aligned}
r_{\mathrm{ER}} & =\frac{M_{\mathrm{cat}}\left(k_{f}^{+} K_{\mathrm{IPA}} a_{\mathrm{HAc}} a_{\mathrm{IPA}}-k_{r}^{-} K_{\mathrm{IPAc}} K_{\mathrm{H}_{2} \mathrm{O}} a_{\mathrm{IPAc}} a_{\mathrm{H}_{2} \mathrm{O}}\right)}{1+K_{\mathrm{IPA}} a_{\mathrm{IPA}}+K_{\mathrm{IPAc}} a_{\mathrm{IPAc}}} \\
& =\frac{M_{\mathrm{cat}}\left(k_{f} a_{\mathrm{HAc}} a_{\mathrm{IPA}}-k_{r} a_{\mathrm{IPAc}} a_{\mathrm{H}_{2} \mathrm{O}}\right)}{1+K_{\mathrm{IPA}} a_{\mathrm{IPA}}+K_{\mathrm{IPAc}} a_{\mathrm{IPAc}}}
\end{aligned}
$$

where $K_{\mathrm{IPA}}$ and $K_{\mathrm{IPAc}}$ respectively, are the adsorption equilibrium constants of IPA and the desorption equilibrium constant of IPAc, $k_{f}^{+}$and $k_{r}^{-}$are the rate constants of forward 
and reverse reactions of surface reactions, and $k_{f}=k_{f}^{+} K_{\mathrm{IPA}}, k_{r}=$ $k_{r}^{-} K_{\mathrm{IPAc}}$ and $K_{\mathrm{a}}=k_{f} / k_{r}$.

The LHHW model assumes that there is only one type of active center on the surface of the catalyst and HAc and IPA undergo competitive adsorption on the active center of cation exchange resin. In addition, each molecule can only be adsorbed on one active center. The surface reaction of HAc and IPA is a rate-controlled step, and the rest of the diffusion, adsorption, and desorption processes are in equilibrium. Thus, the LHHW model can be expressed as follows

$$
\begin{aligned}
& r_{\mathrm{LHHW}}= \\
& \frac{M_{\mathrm{cat}}\left(k_{f}^{+} K_{\mathrm{HAc}} K_{\mathrm{IPA}} a_{\mathrm{HAc}} a_{\mathrm{IPA}}-k_{r}^{-} K_{\mathrm{IPAc}} K_{\mathrm{H}_{2} \mathrm{O}} a_{\mathrm{IPAc}} a_{\mathrm{H}_{2} \mathrm{O}}\right)}{\left(1+K_{\mathrm{HAc}} a_{\mathrm{HAc}}+K_{\mathrm{IPA}} a_{\mathrm{IPA}}+K_{\mathrm{IPAc}} a_{\mathrm{IPAc}}+K_{\mathrm{H}_{2} \mathrm{O}} a_{\mathrm{H} 2 \mathrm{O}}\right)^{2}} \\
& =\frac{M_{\mathrm{cat}}\left(k_{f} a_{\mathrm{HAc}} a_{\mathrm{IPA}}-k_{r} a_{\mathrm{IPAc}} a_{\mathrm{H}_{2} \mathrm{O}}\right)}{\left(1+K_{\mathrm{HAc}} a_{\mathrm{HAc}}+K_{\mathrm{IPA}} a_{\mathrm{IPA}}+K_{\mathrm{IPAc}} a_{\mathrm{IPAc}}+K_{\mathrm{H}_{2} \mathrm{O}} a_{\mathrm{H}} \mathrm{O}\right)^{2}}
\end{aligned}
$$

where $K_{\mathrm{IPA}}$ and $K_{\mathrm{HAc}}$ are the adsorption equilibrium constants of IPA and HAc, respectively, $K_{\mathrm{IPAc}}$ and $K_{\mathrm{H}_{2} \mathrm{O}}$ are the desorption equilibrium constants of IPAc and $\mathrm{H}_{2} \mathrm{O}$, and $k_{f}=$ $k_{f}^{+} K_{\mathrm{IPA}} K_{\mathrm{HAc}}$ and $k_{r}=k_{r}^{-} K_{\mathrm{IPAc}} K_{\mathrm{H}_{2} \mathrm{O}}$.

Kinetic equations eqs $18-20$ are integrated by the method of Runge-Kutta-Fehlberg ${ }^{14,20}$ with the experimental data. The computational procedure is to minimize the sum of residual squares (SRS) ${ }^{21}$ between the experimental conversion of IPA and the calculated values, which is shown as follows

$$
\mathrm{SRS}=\sum\left(x_{\exp }-x_{\text {cat }}\right)^{2}
$$

The kinetic parameters of different models calculated in eqs 18-20 are listed in Table 4. RMSE, the square root of the

Table 4. Rate Constants of Forward and Reverse Reactions

\begin{tabular}{lcccc}
$\begin{array}{c}\text { kinetic } \\
\text { model }\end{array}$ & $T(\mathrm{~K})$ & $\begin{array}{c}k_{f}^{+} \\
\left(\mathrm{L}^{2} /(\mathrm{mol} \cdot \mathrm{g} \cdot \mathrm{min})\right)\end{array}$ & $\begin{array}{c}k_{r}^{-} \\
\left(\mathrm{L}^{2} /(\mathrm{mol} \cdot \mathrm{g} \cdot \mathrm{min})\right)\end{array}$ & $\begin{array}{c}\text { RMSE } \\
(\%)\end{array}$ \\
PH & 333.15 & $5.60 \times 10^{-6}$ & $2.40 \times 10^{-7}$ & 1.61 \\
& 338.15 & $7.50 \times 10^{-6}$ & $3.26 \times 10^{-7}$ & 1.90 \\
& 343.15 & $1.04 \times 10^{-5}$ & $4.57 \times 10^{-7}$ & 2.21 \\
& 348.15 & $1.46 \times 10^{-5}$ & $6.50 \times 10^{-7}$ & 2.34 \\
ER & 353.15 & $2.21 \times 10^{-5}$ & $9.94 \times 10^{-7}$ & 2.45 \\
& 333.15 & $4.01 \times 10^{-5}$ & $1.72 \times 10^{-6}$ & 0.33 \\
& 338.15 & $5.26 \times 10^{-5}$ & $2.28 \times 10^{-6}$ & 0.50 \\
& 343.15 & $7.12 \times 10^{-5}$ & $3.13 \times 10^{-6}$ & 0.60 \\
& 348.15 & $1.01 \times 10^{-4}$ & $4.50 \times 10^{-6}$ & 0.73 \\
LHHW & 353.15 & $1.47 \times 10^{-4}$ & $6.61 \times 10^{-6}$ & 0.80 \\
& 333.15 & $1.24 \times 10^{-3}$ & $5.32 \times 10^{-5}$ & 0.30 \\
& 338.15 & $1.64 \times 10^{-3}$ & $7.12 \times 10^{-5}$ & 0.45 \\
& 343.15 & $2.47 \times 10^{-3}$ & $1.09 \times 10^{-4}$ & 0.54 \\
& 348.15 & $3.18 \times 10^{-3}$ & $1.42 \times 10^{-4}$ & 0.66 \\
& 353.15 & $4.64 \times 10^{-3}$ & $2.09 \times 10^{-4}$ & 0.70 \\
\hline
\end{tabular}

mean square error, ${ }^{22}$ is used to measure the deviation between the experimental values and the calculated values of the conversion of IPA. It can be seen from Table 4 that the RMSE of the LHHW model is least in three models at the same temperature, which indicates that the LHHW model possesses a higher degree of fitting and accuracy. In addition, the difference between the ER model and the LHHW model is extremely small, the mean relative error ${ }^{2,23,24}$ of which, respectively, are 0.40 and $0.37 \%$, while that of $\mathrm{PH}$ model achieves $6.4 \%$. This may indicate that the kinetic process in the IFBR is more suitable to be described by adsorption models.

In addition, Figure 7 is obtained by plotting with the experimental conversion of IPA and the calculated values from

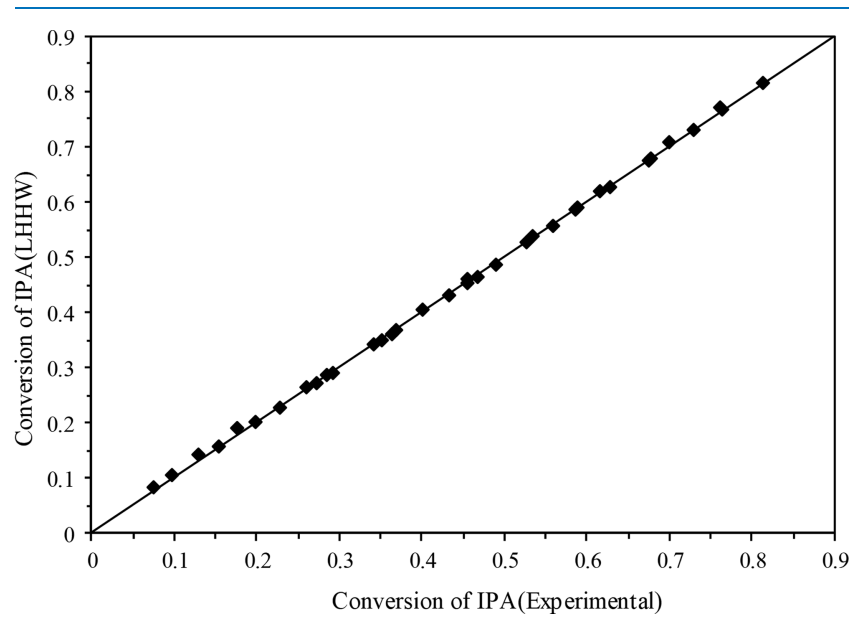

Figure 7. Comparison between experimental and calculated conversions with the LHHW model.

the LHHW model, which can strongly prove the conclusion above. Similarly, figures concerning the ER model and PH model are given in Figures S1 and S2.

$T$ dependence of $k$ can be known from the Arrhenius expression

$$
k=A_{0} e^{-E_{\mathrm{a}} / R T}
$$

which can be changed into

$$
\ln k=\ln A_{0}-\frac{E_{\mathrm{a}}}{R T}
$$

where $A_{0}$ represents the pre-exponential factor, and $E_{\mathrm{a}}$ is the activation energy. The kinetic data of the LHHW model are brought into eq 23 by plotting $\ln k$ against $1 / T$. $T$ dependence of $k$ is given in Figure 8 , and calculated $A_{0}$ and $E_{\mathrm{a}}$ are given in Table 5.

Based on $A_{0}$ and $E_{\mathrm{a}}$ in Table 5, the expressions of forward and reverse reactions are found to be

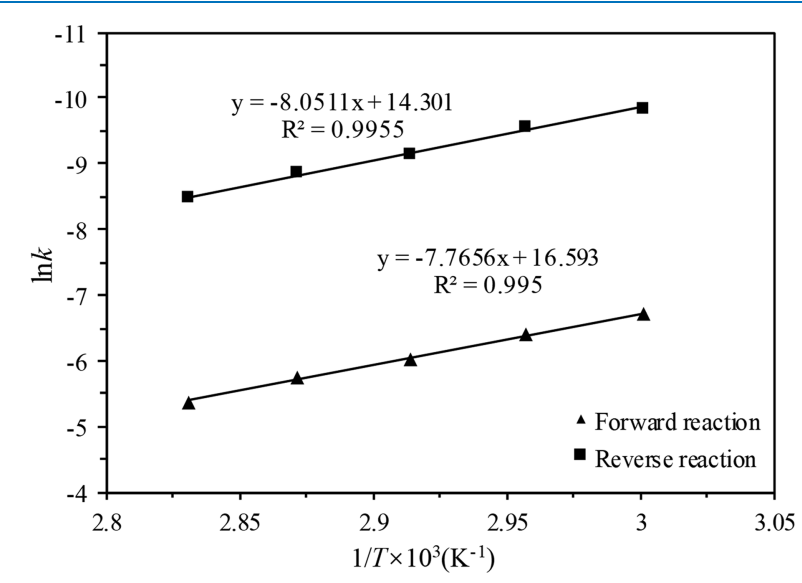

Figure 8. $T$ dependence of $k$. 
Table 5. Pre-Exponential Factors and Activation Energies

\begin{tabular}{ccc} 
LHHW model & $A_{0}\left(\mathrm{~L}^{2} /(\mathrm{mol} \cdot \mathrm{g} \cdot \mathrm{min})\right)$ & $E_{\mathrm{a}}(\mathrm{kJ} / \mathrm{mol})$ \\
forward reaction & $1.608 \times 10^{7}$ & 64.56 \\
reverse reaction & $1.625 \times 10^{6}$ & 66.94 \\
\hline
\end{tabular}

$$
\begin{aligned}
& k_{f}^{+}=1.608 \times 10^{7} \exp \left(-\frac{6.456 \times 10^{4}}{R T}\right) \\
& k_{r}^{-}=1.625 \times 10^{6} \exp \left(-\frac{6.694 \times 10^{4}}{R T}\right)
\end{aligned}
$$

The activation energy for esterification of HAc with IPA catalyzed by Amberlyst 15 in a stirred tank was found to be $60.0 \mathrm{~kJ} / \mathrm{mol}$ by Ali and Merchant. ${ }^{25}$ The similar result, 68.62 $\mathrm{kJ} / \mathrm{mol}$, was obtained by Manning ${ }^{26}$ with the LHHW model. These results are not far from the conclusions in this study.

\section{CONCLUSIONS}

Esterification kinetics of HAc with IPA was studied with Amberlyst 36 Wet in an IFBR at 333-353 K. Effects of different reaction conditions were investigated. $\Delta_{r} H^{0}, \Delta_{r} S^{0}$, and $\Delta_{r} G^{0}$, respectively, were found to be $-2.37 \mathrm{~kJ} / \mathrm{mol}, 19.06 \mathrm{~J} /$ $(\mathrm{mol} \cdot \mathrm{K})$, and $-8.05 \mathrm{~kJ} / \mathrm{mol}(298.15 \mathrm{~K})$ when $\Delta_{r} H^{0}$ was the constant value. The effect of internal diffusion was evaluated by the Weisz-Prater criterion, which was found to be negligible. Three kinds of kinetic models were used to describe the esterification kinetics of HAc with IPA, the results of which showed that adsorption models, especially the LHHW model, were more suitable to explain the esterification reaction process conducted in the IFBR. This provides guidance for industrialized production of IPAc in the fixed bed reactor.

\section{EXPERIMENTAL SECTION}

4.1. Reagents. IPA (analytical reagent (AR), $\geq 99.7 \%$ ) was purchased from Nanjing Chemical Reagent Co., Ltd. HAc (AR, $\geq 99.5 \%$ ) was obtained from Sinopharm Chemical Reagent Co., Ltd. IPAc ( $\geq 99.0 \%)$ was obtained from Energy Chemical. Amberlyst 36 acquired from Sigma-Aldrich is a type of strongly acidic cation exchange resin, the properties of which are shown in Table 6.

\section{Table 6. Properties of Amberlyst 36}

\begin{tabular}{ll}
\multicolumn{1}{c}{ property } & \multicolumn{1}{c}{ Amberlyst 36} \\
matrix & styrene-divinylbenzene \\
standard ionic form & $\mathrm{H}^{+}$ \\
\%moisture & 55 \\
surface area $\left(\mathrm{m}^{2} / \mathrm{g}\right)$ & 33 \\
particle size $(\mathrm{mm})$ & $0.600-0.825$ \\
capacity $($ eq $/ \mathrm{L})$ & $>1.95$ \\
maximum operating temperature $(\mathrm{K})$ & 423 \\
\hline
\end{tabular}

4.2. Apparatus. The reactions were conducted in the IFBR shown in Figure 9, which was equipped with a $250 \mathrm{~mL}$ threenecked glass flask, a high-temperature circulator (298-373 K), a peristaltic pump $(<720 \mathrm{~mL} / \mathrm{min})$, and a stainless steel fixed bed $(1 \mathrm{~cm}$ inner diameter and $30 \mathrm{~cm}$ height $)$. The threenecked glass flask and fixed bed reactor were specially made and both equipped with jackets with the corresponding material for the water bath. The peristaltic pump was obtained from Baoding Lead Fluid Technology Co., LTD, for precise

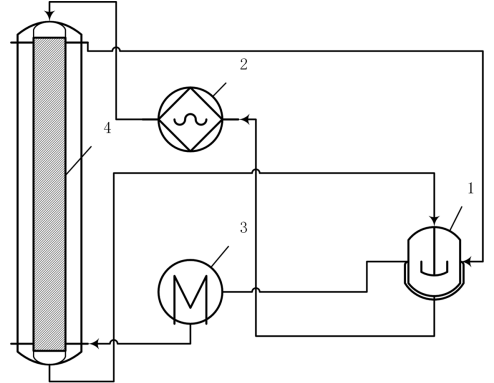

1.preheating tank; 2. peristaltic pump; 3 . high temperature circulator; 4 . fixed bed reactor

Figure 9. Process diagram of IFBR.

control of raw material flow. The high-temperature circulator was equipped with a circulating pump to deliver heat flow to the flask and fixed bed. The system was kept under atmospheric pressure by a cooling coil connected to a neck of the flask.

4.3. Procedure. The fresh catalyst was cleaned with deionized water until the supernatant became achromatic before use. Then, the washed catalyst was soaked in isopropyl alcohol for $24 \mathrm{~h}$, filtered, and dried at $358 \mathrm{~K}$. The prescribed amount of dried catalyst was filled into the fixed bed reactor. $\mathrm{HAc}$ and IPA were added to the $250 \mathrm{~mL}$ three-necked glass flask according to a desired molar ratio, then preheated to the experiment temperature, and stirred with a magnetic stirrer at the same time. The peristaltic pump was opened when the mixture temperature increased to the specified value. Reaction time was recorded at the moment when the mixture entered the fixed bed. Samples were taken from one neck of the flask for gas chromatography analysis at regular intervals after the experiment began.

4.4. Analysis. The composition of raw materials and products was analyzed using a SHIMADZU GC-2014C gas chromatograph, which was equipped with a WondaCap-5 capillary column $(30 \mathrm{~m} \times 0.32 \mathrm{~mm} \times 0.25 \mu \mathrm{m})$ and a flame ionization detector. The temperature of the injection port and detector was set to $240{ }^{\circ} \mathrm{C}$, and the injection volume was 0.5 $\mu \mathrm{L}$. The column temperature was programmed with $40^{\circ} \mathrm{C}$ and initially kept for $4 \mathrm{~min}$, followed by $10^{\circ} \mathrm{C} \cdot \mathrm{min}^{-1}$ rising to 80 ${ }^{\circ} \mathrm{C}$, which was held for $1 \mathrm{~min}$.

\section{ASSOCIATED CONTENT}

\section{Supporting Information}

The Supporting Information is available free of charge on the ACS Publications website at DOI: 10.1021/acsomega.9b02994.

Table S1, value of the volume and area parameters of the groups; Table S2, interaction parameters of the groups $a_{\mathrm{mn}} / K$; Table S3, Weisz-Prater parameters at different temperatures; Figure S1, comparison between experimental and calculated conversions with the $\mathrm{PH}$ model; Figure S2, comparison between experimental and calculated conversions with the ER model (PDF)

\section{AUTHOR INFORMATION}

\section{Corresponding Author}

*E-mail: zhouzheng@nju.edu.cn.

ORCID $\odot$

Ying Liu: 0000-0002-5986-3244 


\section{Notes}

The authors declare no competing financial interest.

\section{ACKNOWLEDGMENTS}

We are particularly grateful to the financial support of National Key Research Program of China (no. 2018YFB0604600-05), Jiangsu Science and Technology Plan Project (no. BM2018007), the Fundamental Research Funds for the Central Universities.

\section{NOMENCLATURE}

$K_{\mathrm{a}} \quad$ reaction equilibrium constant

$a_{i} \quad$ activity of component $i$ at equilibrium

$x_{i} \quad$ mole fraction of component $i$ at equilibrium

$\gamma_{i} \quad$ activity coefficient of component $i$ at equilibrium

$\Delta_{r} H^{0}$ enthalpy $(\mathrm{kJ} / \mathrm{mol})$

$\Delta_{r} S^{0}$ entropy $(\mathrm{J} /(\mathrm{mol} \cdot \mathrm{K}))$

$\Delta_{r} G^{0}$ Gibbs free energy $(\mathrm{kJ} / \mathrm{mol})$

$C_{p, i} \quad$ molar heat capacity of component $i(\mathrm{~J} /(\mathrm{mol} \cdot \mathrm{K}))$

$C_{p}^{0}$ heat capacity of an ideal gas $(\mathrm{J} /(\mathrm{mol} \cdot \mathrm{K}))$

$T_{c} \quad$ critical temperature $(\mathrm{K})$

$T_{r} \quad$ contrast temperature

$C_{\mathrm{wp}} \quad$ Weisz-Prater criterion

$r_{\text {obs }}$ observed reaction rate $(\mathrm{mol} /(\mathrm{L} \cdot \mathrm{min}))$

$\rho_{\mathrm{p}} \quad$ density of catalyst $(\mathrm{g} / \mathrm{mL})$

$R_{c} \quad$ ratio of the volume of catalyst pellet to the external surface area $(\mathrm{cm})$

$D_{\mathrm{e}} \quad$ effective diffusion coefficient $\left(\mathrm{cm}^{2} / \mathrm{s}\right)$

$D_{\mathrm{A}}$ infinite dilution diffusion diffusivity $\left(\mathrm{cm}^{2} / \mathrm{s}\right)$

$\varepsilon_{\mathrm{p}} \quad$ porosity

$\tau \quad$ tortuosity factor

$\mu \quad$ viscosity

$V_{b} \quad$ molar volume at normal boiling temperature $(\mathrm{L} / \mathrm{mol})$

$M_{\text {cat }}$ mass of catalyst per unit volume of the reactants $(\mathrm{g} / \mathrm{L})$

$k_{f} \quad$ apparent forward reaction rate constant $\left(\mathrm{L}^{2} /(\mathrm{mol} \cdot \mathrm{g}\right.$. $\min )$ )

$k_{r} \quad$ apparent reverse reaction rate constant $\left(\mathrm{L}^{2} /(\mathrm{mol} \cdot \mathrm{g}\right.$. $\min )$ )

$k_{f}^{+} \quad$ forward reaction rate constant of surface reaction $\left(\mathrm{L}^{2} /\right.$ $(\mathrm{mol} \cdot \mathrm{g} \cdot \mathrm{min}))$

$k_{r}^{-} \quad$ reverse reaction rate constant of surface reaction $\left(\mathrm{L}^{2} /\right.$ $(\mathrm{mol} \cdot \mathrm{g} \cdot \mathrm{min}))$

$x_{\text {cal }} \quad$ calculated conversion of IPA

$x_{\exp }$ experimental conversion of IPA

$A_{0} \quad$ pre-exponential factor $\left(\mathrm{L}^{2} /(\mathrm{mol} \cdot \mathrm{g} \cdot \mathrm{min})\right)$

$k$ reaction rate constant $\left(\mathrm{L}^{2} /(\mathrm{mol} \cdot \mathrm{g} \cdot \mathrm{min})\right)$

$E_{\mathrm{a}}$ activation energy $(\mathrm{kJ} / \mathrm{mol})$

\section{REFERENCES}

(1) Qi, W.; Malone, M. F. Semibatch reactive distillation for isopropyl acetate synthesis. Ind. Eng. Chem. Res. 2011, 50, 12721277.

(2) Akyalçın, S. Kinetic study of the formation of isopropyl alcohol by transesterification of isopropyl acetate with methanol in the presence of heterogeneous catalyst. Ind. Eng. Chem. Res. 2014, 53, 9631-9637.

(3) Zhang, B. J.; Yang, W. S.; Hu, S.; Liang, Y. Z.; Chen, Q. L. A reactive distillation process with a sidedraw stream to enhance the production of isopropyl acetate. Chem. Eng. Process. 2013, 70, 117130.

(4) Altıokka, M. R.; Çıtak, A. Kinetics study of esterification of acetic acid with isobutanol in the presence of amberlite catalyst. Appl. Catal., A 2003, 239, 141-148.
(5) Osorio-Viana, W.; Duque-Bernal, M.; Fontalvo, J.; DobroszGómez, I.; Gómez-García, M. Á. Kinetic study on the catalytic esterification of acetic acid with isoamyl alcohol over Amberlite IR120. Chem. Eng. Sci. 2013, 101, 755-763.

(6) JagadeeshBabu, P. E.; Sandesh, K.; Saidutta, M. B. Kinetics of esterification of acetic acid with methanol in the presence of ion exchange resin catalysts. Ind. Eng. Chem. Res. 2011, 50, 7155-7160.

(7) Chin, S. Y.; Ahmad, M. A. A.; Kamaruzaman, M. R.; Cheng, C. $\mathrm{K}$. Kinetic studies of the esterification of pure and dilute acrylic acid with 2-ethyl hexanol catalysed by Amberlyst 15. Chem. Eng. Sci. 2015, $129,116-125$.

(8) Akbay, E. Ö.; Altıkka, M. R. Kinetics of esterification of acetic acid with $\mathrm{n}$-amyl alcohol in the presence of Amberlyst-36. Appl. Catal. A 2011, 396, 14-19.

(9) Santacesaria, E.; Tesser, R.; Di Serio, M.; Guida, M.; Gaetano, D.; Garcia Agreda, A. Kinetics and mass transfer of free fatty acids esterification with methanol in a tubular packed bed reactor: a key pretreatment in biodiesel production. Ind. Eng. Chem. Res. 2007, 46, $5113-5121$

(10) Lee, M.-J.; Wu, H.-T.; Lin, H.-m. Kinetics of catalytic esterification of acetic acid and amyl alcohol over Dowex. Ind. Eng. Chem. Res. 2000, 39, 4094-4099.

(11) Cheng, Y.; Feng, Y.; Ren, Y.; Liu, X.; Gao, A.; He, B.; Yan, F.; $\mathrm{Li}$, J. Comprehensive kinetic studies of acidic oil continuous esterification by cation-exchange resin in fixed bed reactors. Bioresour. Technol. 2012, 113, 65-72.

(12) Tsai, Y.-T.; Lin, H.-m.; Lee, M.-J. Kinetics behavior of esterification of acetic acid with methanol over Amberlyst 36. Chem. Eng. J. 2011, 171, 1367-1372.

(13) Ali, S. H. Kinetics of catalytic esterification of propionic acid with different alcohols over Amberlyst 15. Int. J. Chem. Kinet. 2009, 41, 432-448.

(14) He, X.; Xu, R.; Wang, Y.; Zhang, F.; Lei, Y.; Zhou, Z.; Zhang, Z. Methoxylation of dihydromyrcene in an intensified fixed bed reactor. Chem. Eng. Res. Des. 2017, 122, 254-262.

(15) Chakrabarti, A.; Sharma, M. M. Cationic ion exchange resins as catalyst. React. Polym. 1993, 20, 1-45.

(16) Poling, B. E.; Prausnitz, J. M.; O'connell, J. P. The properties of gases and liquids; 5th ed., Mcgraw-hill: New York, 2001.

(17) He, X.; Xu, R.; Zhang, L.; Zhang, F.; Zhou, Z.; Zhang, Z. Alkoxylation of camphene catalyzed by cation exchange resins. Chem. Eng. Res. Des. 2016, 114, 60-68.

(18) Foggler, H. S. Elements of Chemical Reaction Engineering; Prentice Hall: Englewood Cliffs, NJ, USA, 1999.

(19) Wakao, N.; Smith, J. M. Diffusion in catalyst pellets. Chem. Eng. Sci. 1962, 17, 825-834.

(20) Chen, S.; Wang, S.; Zhou, Z.; Yang, G.; Zhang, Z. Study on the Alkoxylation of Dihydromyrcene over Cation Exchange Resins. Ind. Eng. Chem. Res. 2019, 58, 11871-11877.

(21) Silva, V. M. T. M.; Rodrigues, A. E. Kinetic studies in a batch reactor using ion exchange resin catalysts for oxygenates production: Role of mass transfer mechanisms. Chem. Eng. Sci. 2006, 61, 316-331.

(22) Molinero, L.; Ladero, M.; Tamayo, J. J.; García-Ochoa, F. Homogeneous catalytic esterification of glycerol with cinnamic and methoxycinnamic acids to cinnamate glycerides in solventless medium: Kinetic modeling. Chem. Eng. J. 2014, 247, 174-182.

(23) Sert, E.; Buluklu, A. D.; Karakuş, S.; Atalay, F. S. Kinetic study of catalytic esterification of acrylic acid with butanol catalyzed by different ion exchange resins. Chem. Eng. Process. 2013, 73, 23-28.

(24) Kolah, A. K.; Asthana, N. S.; Vu, D. T.; Lira, C. T.; Miller, D. J. Reaction kinetics for the heterogeneously catalyzed esterification of succinic acid with ethanol. Ind. Eng. Chem. Res. 2008, 47, 5313-5317.

(25) Ali, S. H.; Merchant, S. Q. Kinetics of the esterification of acetic acid with 2-propanol: Impact of different acidic cation exchange resins on reaction mechanism. Int. J. Chem. Kinet. 2006, 38, 593-612.

(26) Manning, J. M. Kinetics and Feasiability of Reactive Distillation in Isopropyl Acetate Synthesis; Doctoral dissertation. University of Massachusetts at Amherst, 1999. 